\title{
EVALUACIÓN DE SISTEMAS DE SALUD ANIMAL: DEMANDA DE SERVICIOS EN LA CIUDAD AUTÓNOMA DE BUENOS AIRES
}

\author{
Evaluation of Animal Health Systems: Demand for Services in the \\ Autonomous City of Buenos Aires
}

\author{
María Cecilia Martínez ${ }^{1}$, Analía Tortosa ${ }^{1}$, José Luis Molina ${ }^{1,2}$, Gabriel Pisapía ${ }^{2}$, \\ Edgardo Raúl Marcos ${ }^{1,2}$, Osvaldo Jorge Degregorio ${ }^{1,3}$
}

\section{Resumen}

Se plantea analizar la visión de la comunidad en correspondencia a sus necesidades socio-culturales, evaluando como influye en las decisiones de tenencia de animales domésticos de compañía y en la demanda de los servicios de salud para sus animales. El objetivo de este estudio fue analizar la demanda actual de servicios de salud animal en la Ciudad Autónoma de Buenos Aires. Se realizó un estudio bajo el modelo de encuesta social en un grupo de habitantes que concurrieron a los servicios del Instituto de Zoonosis Dr. Luis Pasteur (IZLP) y en un grupo de comparación en plazas y calles de la ciudad que realizaban actividades no relacionadas con la atención de animales de compañía. No se observaron diferencias en las características demográficas de los grupos en estudio. La atención veterinaria solicitada en el último año fue similar en ambos grupos, aunque variando en los servicios requeridos. Ambos grupos consideraron como satisfactoria las prestaciones de salud pero indicaron la necesidad de incorporar o intensificar acciones de salud, las cuales, en muchos casos, ya estaban en ejecución.

Palabras clave: demanda, evaluación, sistemas de salud animal

\section{Abstract}

The analysis of the vision of the community in line with the socio-cultural needs is proposed in order to assess how influence decisions on the tenure of pets and on the demand for animal health services. The aim of this study was to analyze the current demand for animal health services in the Autonomous City of Buenos Aires. The study used the model of social survey in a group of people who attended the services of the

\footnotetext{
${ }^{1}$ Facultad de Ciencias Veterinarias, Universidad de Buenos Aires, Ciudad Autónoma de Buenos Aires, Argentina

${ }^{2}$ Instituto de Zoonosis Dr. Luis Pasteur, Ciudad Autónoma de Buenos Aires, Argentina

${ }^{3}$ E-mail: odegre@fvet.uba.ar

Recibido: 7 de mayo de 2013

Aceptado para publicación: 3 de julio de 2013
} 
Institute of Zoonosis Dr. Louis Pasteur (IZLP) and a comparison group who performed activities in the city that were unrelated to animal health. There was no difference in the demographic characteristics of the studied groups. Requested veterinary care in the past year was similar in both groups, but differing on the type of services required. Both groups considered as satisfactory the health services but felt the need to incorporate or enhance health actions, which in many cases, were already running.

Key words: demand, evaluation, animal health system

\section{INTRODUCCIÓN}

La estrecha relación y convivencia entre los animales y el hombre en el ámbito urbano se viene incrementando en las últimas décadas, generando bienestar en las personas y en la comunidad, pero por otro lado, creando una fuente de riesgo en la salud pública, debido a la posible transmisión de enfermedades zoonóticas y agresiones, así como afectando el medio ambiente por la eliminación de heces y orina en la vía pública (Degregorio y Sommerfelt, 2007). Por ello, y como parte del estudio de la problemática global, se ha venido estudiando las poblaciones de animales de compañía en relación a los aspectos demográficos (Degregorio et al., 2005) y de su tenencia (Tortosa et al., 2011a), así como las percepciones de los profesionales (Degregorio y Sommerfelt, 2007, 2009) y la influencia de motivaciones, actitudes y conductas de los tenedores de estos animales (Degregorio, 2010; Tortosa et al., 2011b).

Los sistemas locales de salud animal, servicios oficiales que se ofrecen en áreas urbanas, se plantean y desarrollan bajo las premisas de sistemas de vigilancia epidemiológica, permitiendo identificar los problemas relevantes de salud, desarrollando el conocimiento actualizado de los factores epidemiológicos que los condicionan, con la finalidad de intervenir precozmente en la prevención y control de las enfermedades (Betti et al., 2005).

Las percepciones de riesgo de las enfermedades animales son esenciales en la comprensión de las conductas relacionadas con las estrategias para combatir dichas enfermedades. En estudios del impacto de acciones de control de las poblaciones animales, se ha determinado, por ejemplo, que las áreas que detentan mayores índices de esterilización en canes presentan índices de crecimiento similares a áreas con índices de esterilización muy inferiores (Zumpano et al., 2011), demostrando que estos resultados son dependientes de las actitudes y conductas de los tenedores e independientes del estado reproductivo de los animales.

Poco se sabe acerca de los procesos cognitivos subyacentes en la población en relación a sus necesidades y sus percepciones con referencia a los sistemas de salud. La exploración de factores conductuales en tenedores de animales y la comprensión de los citados procesos es crucial para la propuesta e implementación de acciones efectivas frente a las enfermedades (Valeeva et $a l ., 2011)$. Con el fin de aportar conocimientos a los procesos de evaluación de los sistemas de salud animal, se planteó el presente estudio a fin de analizar la demanda actual de servicios de salud animal en la Ciudad Autónoma de Buenos Aires (CABA).

\section{Materiales y Métodos}

El estudio se realizó bajo el modelo de encuesta social, considerando una confianza de $95 \%$ y una precisión de $80 \%$, estimándose un tamaño de muestra $(n=120)$ para demostrar diferencias significativas de al menos 10\% entre dos grupos (Altman, 1991). 
En el transcurso de 2012 se encuestó a un grupo de habitantes de $\mathrm{CABA}$ que concurrieron a los servicios del Instituto de Zoonosis Dr. Luis Pasteur (IZLP), organismo público dependiente del Ministerio de Salud del Gobierno de la Ciudad de Buenos Aires (Grupo 1) y a un grupo de comparación de habitantes que se desplazaban por plazas y calles de la ciudad realizando actividades no relacionadas con la atención médica o el cuidado de animales de compañía (Grupo 2).

Se colectó información demográfica del encuestado (género, edad, tipo de vivienda, conformación del grupo familiar) y de la tenencia de animales (atención veterinaria en el último año y servicios requeridos) de acuerdo a un formulario preestablecido. Asimismo, se consultó acerca de la necesidad de incorporar nuevos servicios. El conocimiento acerca de la oferta de servicios y su valoración fueron evaluados a través de la construcción de la escala Likert, escala semicuantitativa (Degregorio, 2010).

En el desarrollo de la base de datos y su análisis estadístico y epidemiológico se empleó el programa EpiInfo 3.6.1. (CDC/ WHO), software de dominio público. Se empleó la prueba de " $t$ " y su ajuste de varianzas para variables cuantitativas. Se estimaron las proporciones y su intervalo de $95 \%$ de confianza de las variables cualitativas en estudio. Para el análisis estadístico entre grupos se empleó el test de diferencia de proporciones (Fleiss, 1981).

\section{Resultados}

Se realizaron 124 encuestas en el Grupo 1 y 122 en el Grupo 2. Las características de los encuestados, así como la tenencia de animales y su contacto con el instituto en los últimos 12 meses se presentan en el Cuadro 1.
El nivel de conocimientos sobre la existencia del IZLP y el nivel gubernamental del que depende el instituto se presentan en el Cuadro 2. Los servicios de salud más requeridos por los grupos encuestados se presentan en el Cuadro 3. El nivel de satisfacción adecuado (muy satisfactorio y satisfactorio) para el total de servicios fue de 93.0 y $97.1 \%$ para los grupos 1 y 2 , respectivamente (Cuadro 4); sin embargo, el $38.7 \%$ (48/124) del grupo 1 y el $36.0 \%$ (44/122) del grupo 2 consideraron que el instituto debería realizar actividades adicionales, entre estas, la captura y esterilización de animales (grupos 1 y 2) y atención hospitalaria (grupo 1) (Cuadro 5).

\section{Discusión}

No hubo diferencia estadística entre los dos grupos de encuestados en relación a su distribución por género y edad, proporción de personas que viven en CABA, en casas o departamentos, ni en la composición del grupo familiar o en la proporción de personas que viven solas. Sin embargo, la tenencia de animales fue significativamente mayor en el grupo 1 ( $\mathrm{p}<0.01$ ), toda vez que es una muestra sesgada por selección al concurrir al servicio de salud objeto de evaluación; no obstante, la tenencia en el grupo 2 no difiere de la estimada en CABA (Degregorio, 2010), lo cual fundamenta la comparabilidad de los resultados del presente trabajo.

No se observaron diferencias significativas entre los grupos en estudio en cuanto a la atención de los animales en los últimos 12 meses; sin embargo, cabe considerar que si bien el Grupo 1 lo hizo a través del instituto, el $72.5 \%$ lo hizo no solo a través del IZLP sino, además, con otros prestadores de servicio privados o públicos de salud.

La demanda de servicios de salud fue diferente entre los dos grupos. Los encuestados en el servicio de salud público (Grupo 1) requirieron principalmente atención 
Cuadro 1. Encuesta sobre demanda de servicios de salud animal a habitantes de la Ciudad Autónoma de Buenos Aires que concurrieron a los servicios del Instituto de Zoonosis Dr. Luis Pasteur (grupo 1) o que se encontraban realizando otras actividades (grupo 2)

\begin{tabular}{lcc}
\hline Variables & $\begin{array}{c}\text { Grupo } 1 \\
(\mathrm{n}=124)\end{array}$ & $\begin{array}{c}\text { Grupo } 2 \\
(\mathrm{n}=122)\end{array}$ \\
\hline Género (\%) & $50(40.3 \%)$ & $64(52.5 \%)$ \\
$\quad$ Masculino & $74(59.7 \%)$ & $58(47.5 \%)$ \\
$\quad$ Femenino & & \\
Edad (años) & $42.2 \pm 14.0$ & $43.1 \pm 15.6$ \\
$\quad$ Masculino & $45.1 \pm 16.2$ & $39.5 \pm 13.7$ \\
$\quad$ Femenino & $87.1 \%(108 / 124)$ & $79.5 \%(97 / 122)$ \\
Vive en CABA & $42.6 \%(46 / 108)$ & $32.0 \%(31 / 97)$ \\
$\quad$ En casa & $57.4 \%(62 / 108)$ & $68.0 \%(66 / 97)$ \\
$\quad$ En departamento & $14.8 \%(16 / 108)$ & $22.7 \%(22 / 97)$ \\
Vive solo & $3.4 \pm 2.0$ & $3.0 \pm 2.1$ \\
Grupo familiar $\left(\mathrm{N}^{\circ}{ }^{\circ}\right.$ de & $97.6 \%(121 / 124)$ & $65.6 \%(80 / 122)$ \\
personas) & $81.0 \%(98 / 121)$ & $40.0 \%(32 / 80)^{2}$ \\
Tiene animales &
\end{tabular}

${ }^{1}$ Recibieron atención en el IZLP en los últimos 12 meses.

${ }^{2} 72.5 \%$ (58/80) considerando prestadores de servicios privados o públicos de salud

Cuadro 2. Nivel de conocimientos de los encuestados sobre la existencia del Instituto de Zoonosis Dr. Luis Pasteur (IZLP) y el nivel gubernamental del que depende el instituto $^{1}$

\begin{tabular}{lcc}
\hline Variable de conocimiento & $\begin{array}{c}\text { Clientes de servicios } \\
\text { del IZLP }\end{array}$ & $\begin{array}{c}\text { Encuestados en } \\
\text { plazas y calles }\end{array}$ \\
\hline $\begin{array}{l}\text { Dice conocer la existencia del IZLP } \\
\text { Dice conocer el nivel gubernamental que } \\
\text { depende el IZLP }\end{array}$ & $97.6 \%(121 / 124)$ & $75.4 \%(92 / 122)$ \\
Respuesta al nivel gubernamental del IZLP & $80.2 \%(97 / 121)$ & $81.5 \%(75 / 92)$ \\
$\quad$ Nivel municipal & $55.7 \%(54 / 97)$ & $49.3 \%(37 / 75)$ \\
$\quad$ Nivel nacional & $27.8 \%(27 / 97)$ & $37.3 \%(28 / 75)$ \\
$\quad$ Universidad de Buenos Aires ${ }^{2}$ & $5.1 \%(5 / 97)$ & $6.7 \%(5 / 75)$ \\
$\quad$ Otros niveles de gobierno & $11.4 \%(11 / 97)$ & $6.7 \%(5 / 75)$ \\
\hline
\end{tabular}

${ }_{2}^{1}$ Dependencia del Ministerio de Salud del Gobierno de la Ciudad de Buenos Aires

${ }^{2}$ Facultad de Ciencias Veterinarias 
Cuadro 3. Servicios con mayor demanda dentro del sistema de salud animal por los grupos encuestados

\begin{tabular}{lcc}
\hline \multirow{2}{*}{ Servicios } & $\begin{array}{c}\text { Clientes de servicios del } \\
\text { IZLP }\end{array}$ & $\begin{array}{c}\text { Encuestados en plazas } \\
\text { y calles }\end{array}$ \\
\hline Consultorio clínico & $55.9 \%$ & $40.8 \%$ \\
Esterilización quirúrgica & $48.3 \%$ & $56.3 \%$ \\
Inmunizaciones & $16.1 \%$ & $4.2 \%$ \\
Atención de agresiones & $11.0 \%$ & $43.7 \%$ \\
Adopciones & $2.5 \%$ & $2.8 \%$ \\
\hline
\end{tabular}

Cuadro 4. Satisfacción de servicios de salud animal prestados por el Instituto de Zoonosis Dr. Luis Pasteur (2012) - Expresión de respuestas múltiples

\begin{tabular}{llcccc}
\hline & & \multicolumn{4}{c}{ Nivel de satisfacción } \\
\cline { 3 - 6 } & $\begin{array}{c}\text { Muy } \\
\text { satisfecho }\end{array}$ & Satisfecho & $\begin{array}{c}\text { Poco } \\
\text { satisfecho }\end{array}$ & $\begin{array}{c}\text { No } \\
\text { satisfecho }\end{array}$ \\
\hline Grupo 1 & Consultorio clínico & 29 & 32 & 2 & 3 \\
& Esterilización quirúrgica & 22 & 30 & 3 & 2 \\
& Inmunizaciones & 8 & 10 & 1 & - \\
& Atención de agresiones & 6 & 7 & - & - \\
& Adopciones & 2 & 1 & - & - \\
Grupo 2 & Consultorio clínico & 10 & 17 & 1 & 1 \\
& Esterilización quirúrgica & 18 & 22 & - & - \\
& Inmunizaciones & 1 & 2 & - & - \\
& Atención de agresiones & 14 & 16 & 1 & - \\
& Adopciones & - & 2 & - & - \\
\hline
\end{tabular}

${ }^{1}$ Grupo 1: La encuesta se hizo a personas que concurrieron a los servicios del IZLP; Grupo 2: Se hizo a personas en plazas y calles de la ciudad

en consultorios y esterilización quirúrgica, mientras que el grupo general (Grupo 2) requirió servicios de esterilización quirúrgica, atención de agresiones y atención en consultorios. Fue evidente que la adopción de animales resultó el servicio menos requerido por ambos grupos.
Respecto al elevado registro de consultas por agresiones observado entre los encuestados del grupo 2 (43.7\%, Cuadro 3 ), cabe aclarar que una de las funciones históricas más importantes del IZLP ha sido, justamente, la recepción de denuncias por agresiones de animales. En los periodos en los 
Cuadro 5. Sugerencias de ampliación de servicios del Instituto de Zoonosis Dr. Luis Pasteur por parte de los encuestados

\begin{tabular}{lcccc}
\hline \multirow{2}{*}{ Actividades } & \multicolumn{2}{c}{$\begin{array}{c}\text { Clientes de } \\
\text { servicios del IZLP } \\
(\mathrm{n}=48)\end{array}$} & $\begin{array}{c}\text { Encuestados en } \\
\text { plazas y calles } \\
(\mathrm{n}=44)\end{array}$ \\
\cline { 2 - 6 } & $\mathrm{n}$ & $\%$ & $\mathrm{n}$ & $\%$ \\
\hline Captura y esterilización de animales & 11 & 22.9 & 20 & 45.5 \\
Atención hospitalaria & 10 & 20.8 & - & - \\
Más personal / Turnos electrónicos o telefónicos & 7 & 14.6 & - & - \\
Campañas en tenencia responsable de animales & 5 & 10.4 & 6 & 13.6 \\
Crear una cooperativa & 5 & 10.4 & - & - \\
Mayor difusión de las ac tividades del IZLP & 4 & 8.3 & 8 & 18.2 \\
\hline
\end{tabular}

cuales había casos de rabia canina en la CABA, se alcanzaron picos de hasta 15000 denuncias por año, lo cual determinó que el servicio de recepción de este tipo de eventos se mantuviese abierto todos los días del año, incluyendo domingos y feriados. El promedio de denuncias en los últimos años se encuentra estabilizado en cerca de 8000 por año, es decir, 22 denuncias/día, el cual es un valor similar al de accidentes viales en la CABA. Esto da una idea de la importancia que adquiere el evento «agresiones animales», especialmente si se considera que solo la mitad de las agresiones producidas por animales son denunciadas.

La valoración de las acciones del servicio de salud animal local fue satisfactoria, aunque se debe resaltar que cerca de la mitad de las personas de ambos grupos desconocía la relación administrativa existente entre el IZLP y el Ministerio de Salud del Gobierno de la Ciudad de Buenos Aires. Asimismo, ambos grupos consideraron la necesidad de incorporar o intensificar acciones de salud, que en muchos casos se encuentran en ejecución, lo cual cabe reflexionar si es un problema de desconocimiento por parte de los beneficiarios del servicio, en particular cuando solicitan atención médica, captura y esterilización o campañas de tenencia o es el reflejo de una deficiente comunicación institución-beneficiarios.

Si bien el IZLP nunca tuvo funciones de hospital veterinario, el imaginario popular sigue prolongando en el tiempo el error de considerarlo como tal. Históricamente, esta institución, creada en 1886, tuvo y tiene como tarea sanitaria resguardar la salud de los habitantes de la CABA frente a las zoonosis. El instituto cuenta con quirófanos fijos y móviles para colaborar en el control poblacional de caninos y felinos, y con consultorios para el diagnóstico clínico y diferencial de las patologías zoonóticas con aquellas que son propias de los animales y no comprometen la salud humana. Estas dos actividades son las que generan cierto grado de confusión en las personas que acuden al instituto a recabar servicios veterinarios y desconocen el resto de las acciones que lleva adelante esta institución.

La evaluación de la demanda de los servicios de salud animal provee una rica información que puede analizarse desde diversos puntos de vista. En primera instancia, per- 
mitiría tener una estimación más certera de los recursos necesarios para atender los problemas de salud en la comunidad. A la vez, el conocimiento del nivel de satisfacción de los demandantes es un claro indicador de calidad del servicio.

\section{Agradecimiento}

Los autores agradecen al personal del Instituto de Zoonosis Dr. Luis Pasteur de la Ciudad Autónoma de Buenos Aires, Argentina por las facilidades prestadas para el desarollo del estudio.

\section{Literatura Citada}

1. Altman DG. 1991. Practical statistics for medical research. London: Chapman y Hall. 900 p.

2. Betti A, Trinidad JJ, Degregorio OJ. 2005. Evaluación sistemas de vigilancia epidemiológica. Vet Argentina XXIII(222): 92-101.

3. Degregorio OJ. 2010. Poblaciones de caninos en áreas urbanas: modelos epidemiológicos. Tesis Doctoral. Argentina: Universidad de Buenos Aires. 120 p.

4. Degregorio OJ, Sommerfelt IE. 2007. Percepciones que tienen los médicos veterinarios en relación con la tenencia de animales de compañía en la ciudad de Buenos Aires. Rev Med Vet 88: 144-148.

5. Degregorio OJ, Sommerfelt IE. 2009. Percepciones que tienen los médicos ve- terinarios en relación con la tenencia de felinos en la ciudad de Buenos Aires. Rev Inv Vet Perú 20: 221-227.

6. Degregorio OJ, López C, Sommerfelt IE. 2005. Tenencia de animales de compañía. Rev Med Vet 86: 141-145.

7. Fleiss JL. 1981. Statistical methods for rates and proportions. $2^{\text {nd }}$ ed. New York: J Wiley \& Sons. 750 p.

8. Tortosa A, Zumpano $R$, Marcos $E$, Degregorio OJ. 2011a. Convivencia humano-animal en áreas urbanas: perfiles de tenencia de caninos en residentes de la ciudad de Buenos Aires. En: VII Jornadas Internacionales de Salud Pública. Argentina: Universidad Nacional de Córdoba.

9. Tortosa A, Zumpano $R$, Marcos $E$, Degregorio OJ. 2011b. Actitudes y conductas en relación con la tenencia de animales de compañía en la ciudad de Buenos Aires. En: VII Jornadas Internacionales de Veterinaria Práctica. Mar de Plata, Argentina.

10. Valeeva NI, Van Asseldonk M, Backus GBC. 2011. Perceived risk and strategy efficacy as motivators of risk management strategy adoption to prevent animal diseases in pig farming. Prev Vet Med 102: 284-295.

11. Zumpano R, Tortosa A, Degregorio OJ. 2011. Estimación del impacto de la esterilización en el índice de crecimiento de la población de caninos. Rev Inv Vet Perú 22: 336-341. 